This item was submitted to Loughborough's Research Repository by the author.

Items in Figshare are protected by copyright, with all rights reserved, unless otherwise indicated.

\title{
Asymmetry of the latent heat signature in b-axis oriented single crystal Gd Si
}

$\mathrm{Ge}$

PLEASE CITE THE PUBLISHED VERSION

http://dx.doi.org/10.1557/opl.2011.617

PUBLISHER

Cambridge University Press (@ Materials Research Society)

VERSION

AM (Accepted Manuscript)

LICENCE

CC BY-NC-ND 4.0

\section{REPOSITORY RECORD}

Morrison, Kelly, V.K. Pecharsky, K.A. Gschneidner, and L.F. Cohen. 2019. "Asymmetry of the Latent Heat Signature in B-axis Oriented Single Crystal Gd Si Ge". figshare. https://hdl.handle.net/2134/12667. 
This item was submitted to Loughborough's Institutional Repository (https://dspace.lboro.ac.uk/) by the author and is made available under the following Creative Commons Licence conditions.

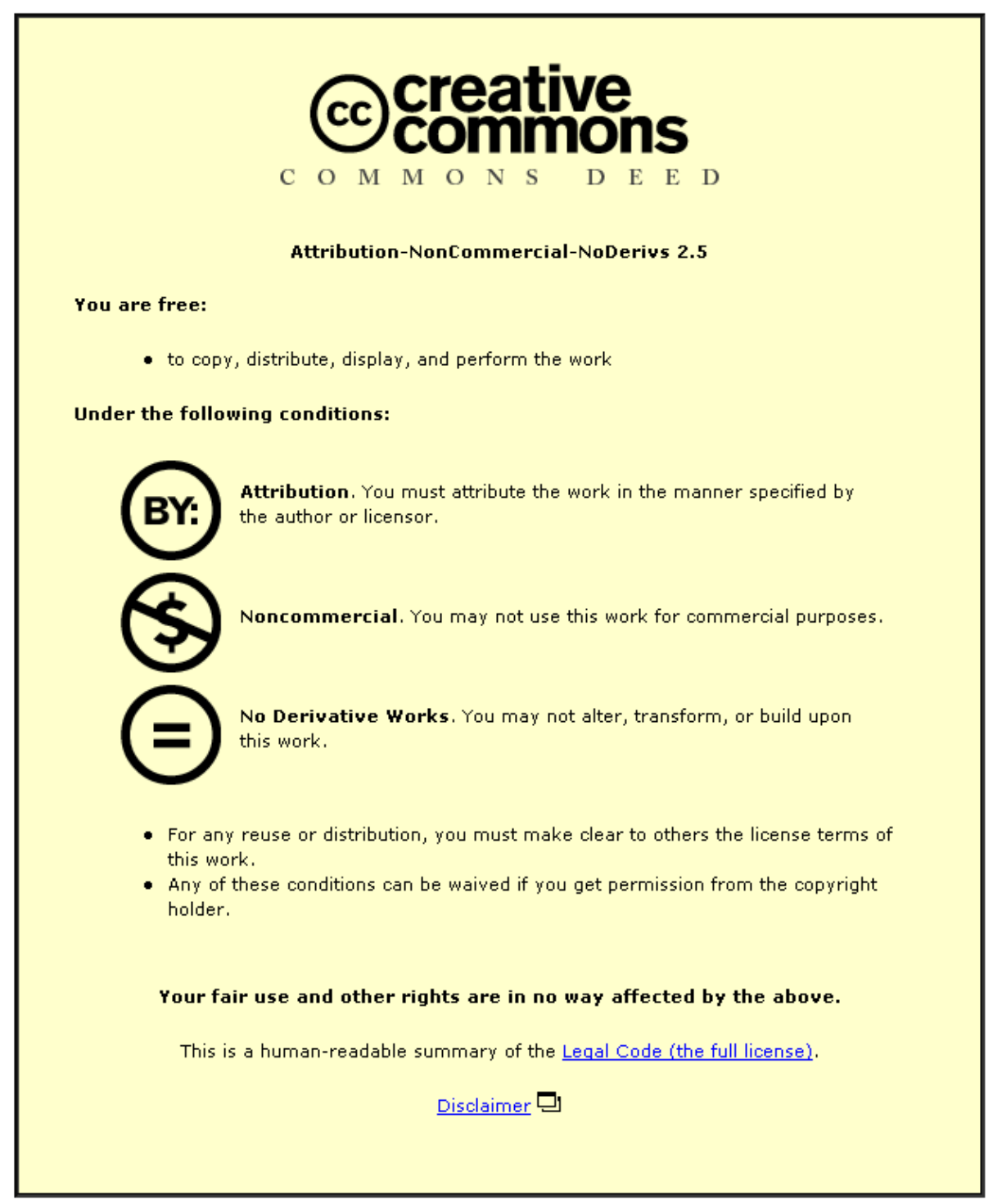

For the full text of this licence, please go to: http://creativecommons.org/licenses/by-nc-nd/2.5/ 


\title{
Asymmetry of the latent heat signature in $b$-axis oriented single crystal $\mathbf{G d}_{5} \mathrm{Si}_{2} \mathbf{G e}_{2}$
}

\author{
K. Morrison ${ }^{1}$, V.K. Pecharsky ${ }^{2}$, K.A. Gschneidner, Jr. ${ }^{2}$, and L.F. Cohen ${ }^{1}$ \\ ${ }^{1}$ Blackett Laboratory, Prince Consort Rd, London SW7 2BZ, UK \\ ${ }^{2}$ Ames Laboratory of the U.S. Department of Energy, and Department of Materials Science and \\ Engineering, Iowa State University, Ames, Iowa, 50011-3020, USA
}

\begin{abstract}
A 100 micron fragment of a $b$-axis oriented single crystal $\mathrm{Gd}_{5} \mathrm{Si}_{2} \mathrm{Ge}_{2}$ has been studied using microcalorimetry, enabling the separate measurement of the heat capacity and the latent heat. The sample was taken from the same crystal previously studied with Hall probe imaging, which showed that the phase transition is seeded by a second phase of $\mathrm{Gd}_{5} \mathrm{Si}_{1.5} \mathrm{Ge}_{1.5}$ nanoplatelets on the increasing field sweep direction only. The multiple transition features observed in the latent heat signature suggests a nucleation size of approximately $20 \mu \mathrm{m}$, consistent with the lengthscale suggested by Hall imaging. The difference in nucleation and growth process with field sweep direction is clearly identified in the latent heat. We show that the latent heat contribution to the entropy change is of the order of $50 \%$ of the total entropy change and unlike other systems studied, the transition does not broaden (and the latent heat contribution does not diminish significantly) as magnetic field and temperature are increased within the parameter range explored in these experiments.
\end{abstract}

\section{INTRODUCTION}

The magnetocaloric effect is observed as a change in temperature of a magnetic material in response to magnetic field; the result of conservation of entropy. Typically large changes in entropy occur when there is a first order phase transition, and this is often found in materials that exhibit a coincident structural phase transition or volume change. Associated with these large entropy changes is hysteresis, $\Delta \mathrm{H}$; a property that may limit the efficiency of the refrigerative cycle. A better understanding of the metamagnetic transitions and how they may be controlled to reduce the critical field, $\mathrm{H}_{\mathrm{c}}$, or $\Delta \mathrm{H}$, are important research directions. Here we relate the different nucleation and growth processes observed previously by Hall probe imaging to their manifestation in latent heat measurements. We also demonstrate that unlike previously studied systems such as $\mathrm{CoMnSi}$ or $\mathrm{La}(\mathrm{Fe}, \mathrm{Si})_{13}$ the latent heat magnitude does not vary significantly with the critical field.

The giant magnetocaloric effect was first reported in $\mathrm{Gd}_{5} \mathrm{Si}_{2} \mathrm{Ge}_{2}$ in $1997^{1}$ and explained as the result of a co-incident orthorhombic (ferromagnetic FM) to monoclinic (paramagnetic PM) structural and magnetic phase transitions. ${ }^{2}$ For the $\mathrm{Gd}_{5} \mathrm{Si}_{2} \mathrm{Ge}_{2}$ composition the $\mathrm{T}_{\mathrm{c}}$ is $274 \mathrm{~K}$. By varying the $\mathrm{Si}$ :Ge content, the $\mathrm{T}_{\mathrm{c}}$ can easily be tuned and the phase transition moves from first to second order as the structural phase transition disappears. ${ }^{3}$

Studies suggest that the structural contribution to entropy change, $\Delta \mathrm{S}_{\text {str }}$, is of the order of $40-60 \%$ of the total entropy change, $\Delta \mathrm{S}_{\text {Total. }}{ }^{4,5,6}$ We use here the separation of the latent heat and the heat capacity terms to probe the first order characteristics of the field driven phase transition in single crystal $\mathrm{Gd}_{5} \mathrm{Si}_{2} \mathrm{Ge}_{2}$ with the field applied along the $b$-axis. 


\section{EXPERIMENT}

Single-crystal samples of $\mathrm{Gd}_{5} \mathrm{Si}_{2} \mathrm{Ge}_{2}$ were prepared as described in References [7,8]. The orientation of the three different crystal faces was established using the back scattered Laue technique and subsequent X-ray diffractometry. The heat capacity and latent heat measurements were carried out using a Xensor Integration SiNi membrane sensor (TCG-3880), adapted for use as an insert for an Oxford Instruments $8 \mathrm{~T}$ magnet and cryostat. The sample is typically a $50 \times 100 \times 100 \mu \mathrm{m}$ fragment; limited by the size of the heater region on the SiN membrane of $50 \times 100 \mu \mathrm{m}$.

For the heat capacity measurements, an ac current is applied to the resistive heater onto which the sample is mounted, and the corresponding temperature modulation is measured by a series of thermocouples. It has been shown that helium gas acting as a heat exchange medium is ideal for utilizing this gauge for ac calorimetry. Due to the hysteretic nature of the first order phase transition, the ac heat capacity method does not fully capture the latent heat. ${ }^{9}$ In the single thermal length limit and treating the heater as a point source, the heat capacity $\mathrm{C}$ is measured from the complex voltage of the thermopile as defined in equation 1 ,

$$
\mathrm{V}_{\mathrm{ac}}=\mathrm{KP} /(i \omega \mathrm{C}+\mathrm{G})
$$

where $P$ is the heater power, $K$ is the Seebeck coefficient $(\mathrm{dV} / \mathrm{dT}), \omega$ is the frequency of temperature modulation (at twice the frequency of the ac current), $G$ is the heat exchange coefficient, ${ }^{11}$ and $\mathrm{C}$ includes the addenda contributions which have to be evaluated separately. ${ }^{10}$

To measure the latent heat as a function of field at constant temperature the helium exchange gas used to set the measurement temperature is pumped out to minimize heat loss to the environment (similar to the method used for differential scanning calorimetry (DSC)). ${ }^{11}$ The calibration is performed by comparison the voltage generated due to latent heat, $\mathrm{V}_{\text {th }}$, to the response, $\mathrm{V}_{\text {cal }}$, to an electrical pulse of known energy content, $\mathrm{E}_{\text {cal }}$.

The entropy change due to changes in the equilibrium heat capacity values, $\Delta S_{H C}$, is evaluated using equation 2 , where $\mathrm{T} 1$ is a reference temperature used for integration and is defined as the temperature at which $\Delta S_{\text {Total }}$ is zero. Note that the \pm sign is dependent on whether $\mathrm{T} 1<\mathrm{T}(+)$ or $\mathrm{T} 1>\mathrm{T}(-)$. The latent heat contribution, $\Delta S_{L H}$, is determined using equation 3 , where $\Delta \mathrm{Q}_{\mathrm{L}}$ is the total heat released by the material. As shown by Pecharsky et al., (2001), ${ }^{12}$ the total entropy change, $\Delta S_{\text {Total }}$, is a sum of the two components $\Delta S_{H \mathrm{C}}$ and $\Delta S_{L H}$.

$$
\begin{aligned}
& \Delta S_{H C}(T)= \pm \int_{T}^{T 1} \frac{(C(H, T)-C(0, T)) d T}{T} \\
& \Delta S_{L H}(T)=-\frac{\Delta Q_{L}}{T}=\frac{V_{t h} E_{\text {cal }}}{V_{\text {cal }}} \\
& \Delta S_{\text {Total }}(T)=\Delta S_{H C}(T)+\Delta S_{L H}(T)
\end{aligned}
$$

\section{EVOLUTION OF THE PHASE TRANSITION}

Before studying the microcalorimetric response it is useful to review the evolution of the phase transition in first order metamagnetic systems in general and also what we have already learnt about $\mathrm{Gd}_{5} \mathrm{Si}_{2} \mathrm{Ge}_{2}$ in particular. 
The mechanism of the field driven phase transition has been demonstrated using Hall probe imaging for several systems. ${ }^{13,14,15}$ For many first order systems, the phase transition evolves via the nucleation and growth type mechanism. ${ }^{16}$ The energy barrier associated with the first order phase transition can result in nucleation of the FM within a PM matrix, brought about by local strain fields or inhomogeneity that (locally) lowers the energy barrier. The balance between the interfacial energies and the energy barrier of the phase transition itself determines the size of the nucleated region. In some systems, the nucleation size will be limited to the grain size and large energy barriers will limit the growth process, described as athermal behavior. ${ }^{17}$ In others the nucleation and growth mechanism will be influenced by thermal activation. ${ }^{13,14}$

For example, extensive studies of the athermal behavior of martensitic phase transitions have been carried out by acoustic emission, differential scanning calorimetry and optical microscopy. ${ }^{18}$ It is a useful system to draw parallels with, as, like $\mathrm{Gd}_{5} \mathrm{Ge}_{2} \mathrm{Si}_{2}$, the structural phase transition occurs by a shearing mechanism. ${ }^{3}$ The coupling of magnetic and structural phase transitions is a result of long-range interactions and as such will involve large energy barriers. Such large energy barriers inevitably result in athermal behavior as the kinetics of the phase transition are more likely to be influenced by changes in the energy landscape brought about by magnetic field or temperature. Dislocations, differences in stoichiometry, grain boundaries etc. also termed here as 'disorder' will act as nucleation centers and encourage metastable states in the macroscopic system by distributing the local onset fields across the bulk sample, resulting in avalanche type behavior.

The dynamics of the first order phase transition as observed by Hall probe imaging has been discussed previously by Perkins et al., with respect to nucleation rate, $R_{N}$, and growth rate, $\mathrm{R}_{\mathrm{G}} .{ }^{16}$ Simple modeling showed that for $\mathrm{R}_{\mathrm{G}}>>\mathrm{R}_{\mathrm{N}}$ the characteristic cluster size, $\mathrm{L}_{\mathrm{C}}$, in a macroscopic system will be large. It was also shown that for $\mathrm{R}_{\mathrm{N}}$ larger than $\mathrm{R}_{\mathrm{G}}$ the characteristic lengthscale will decrease. Here we show results from a microscopic $(100 \mu \mathrm{m})$ system.

It was shown previously by Hall probe imaging that in the $b$-axis orientation the crystal exhibits preferred nucleation on the increasing field sweep only, due to inclusions of nanoplatelet second phase $\mathrm{Gd}_{5} \mathrm{Si}_{1.5} \mathrm{Ge}_{1.5}$ that provides some strain release. The imaging demonstrated that there is an initial nucleation along the nanoplatelets, and from these nano-dimensional seeds the transition spreads through a growth dominated process over the rest of the crystal (with growth rate, $\mathrm{R}_{\mathrm{G}}$ ) resulting in a broad phase transition. On the field decrease the phase transition is not enabled by the nanoplatelets and instead it occurs by large areas of the order of $0.1 \times 0.1 \mathrm{~mm}$ dimension, switching sharply from the FM to the PM phase. In decreasing fields therefore we can consider the transition as nucleation dominated (where each of the large areas are effectively a macroscopic nucleation site) and there is no evidence of growth of the PM phase from the FM phase within the timescales of the image taking process. Hence $\mathrm{R}_{\mathrm{G}}$ is affected in this system by the introduction of nucleation centers, facilitated by the nanoplatelets of $\mathrm{Gd}_{5} \mathrm{Si}_{1.5} \mathrm{Ge}_{1.5}$ on the field increase sweep.

\section{RESULTS AND DISCUSSION}

Figure 1(a)\&(b) shows the results from microcalorimetry for the single crystal $\mathrm{Gd}_{5} \mathrm{Si}_{2} \mathrm{Ge}_{2}$. Notice that the change in heat capacity is step-like; a typical feature of this system ${ }^{19}$ and other first order phase transitions. The latent heat is also shown for field sweep up and down, where it might, on first inspection, appear asymmetric. Unlike previous latent heat measurements, where 
the magnitude of the latent heat spike could be added to give the total, $\Delta \mathrm{Q}_{\mathrm{L}}$, we argue here that the combination of the nucleation and growth mechanism requires different treatment. Note that due to experimental constraints of these microgram samples at high temperatures there can be significant error in the absolute heat capacity. The relative changes, on the other hand are accurate to within $1 \%$ and for this reason we show the sample heat capacity, $\mathrm{C}_{\text {est }}$, as estimated by fitting $\mathrm{C}(\mathrm{H}=0)$ to bulk measurements.

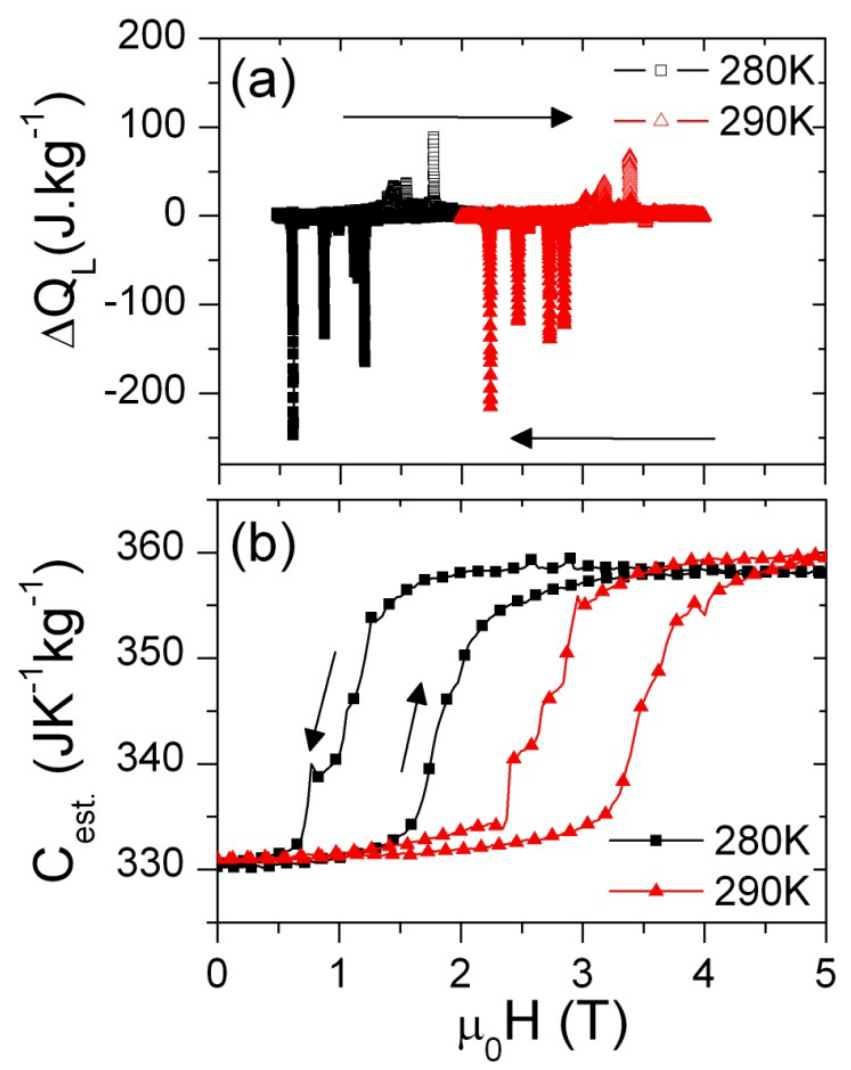

Figure 1. (a) \& (b) Example of the latent heat (a) and the heat capacity (b) of $\mathrm{Gd}_{5} \mathrm{Si}_{2} \mathrm{Ge}_{2}$ single crystal with field applied along the $b$-axis. Arrows indicate the direction of the magnetic field sweep.

The manifestation of the latent heat can follow one of three cases in this measurement, as depicted in figure 2 , where $\xi$ is defined here as the correlation length, equivalent to the characteristic cluster length, $\mathrm{L}_{\mathrm{C}}$, as described in reference [16]. If $\xi$ is of the order of the fragment measured, i.e. $100 \mu \mathrm{m}$, the observed latent heat will be a large jump in $\mathrm{V}_{\text {th }}$, which then decays exponentially with a thermal time constant $\tau_{\text {th }} \sim 1 \mathrm{~s}$. 


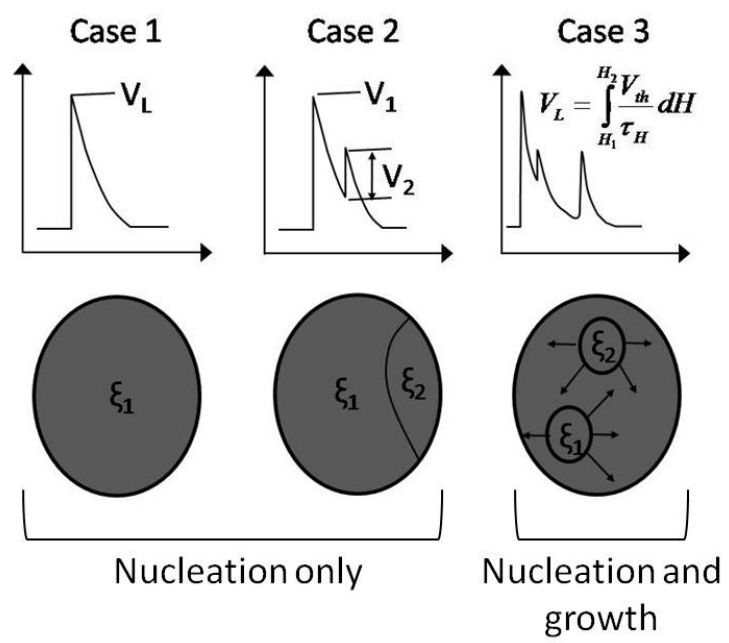

Figure 2. Different manifestations of the latent heat. For correlation length, $\xi \geq 100 \mu \mathrm{m}$, case 1: a single latent heat spike is observed, as was shown in figure 1 . For $\xi<100 \mu \mathrm{m}$, cases $2 \& 3$ : it will be seen that one is nucleation dominated (case 2) and the other is growth dominated (case 3) as explained further in the text.

If $\xi$ is $<100 \mu \mathrm{m}$ there are two processes that can be observed in the latent heat measurement, which is dependent on the relative magnitude $R_{G} / R_{N}$. In case 2 the first order phase transition is nucleation dominated $\left(\mathrm{R}_{\mathrm{G}}<\mathrm{R}_{\mathrm{N}}\right)$, i.e. the energy barrier is large enough that nucleation of distinct separate sites occurs with little or no growth of the FM phase. In case 3 $\left(\mathrm{R}_{\mathrm{G}}>\mathrm{R}_{\mathrm{N}}\right)$, there is significant growth of the FM phase about nucleated sites. As individual latent heat spikes can no longer be identified the total $\Delta \mathrm{Q}_{\mathrm{L}}$ can only be assessed by integration of the total $\mathrm{V}_{\text {th }}$ as described by equation 5 ,

$V_{L}=\int_{H 1}^{H 2} \frac{V_{t h}}{\tau_{H}} d H$

where $\tau_{\mathrm{H}}$ is a time constant determined by the field sweep rate used for the measurement and the thermal time constant $\tau_{\text {th }}$.

Figure $3(\mathrm{a}) \&(\mathrm{~b})$ shows a close-up of the latent heat data at $285 \mathrm{~K}$ demonstrating the impact of the growth dominated process. For the field increase there is a more distributed latent heat response with respect to field, i.e. a bump like feature (b) rather than sharp (a) peaks. The plates which seed the FM phase transition on the increasing field sweep are of the order of 0.2 $\mu \mathrm{m}$ thick, so it is reasonable to assume that a few platelets cross the small sample volume. After the FM phase has been seeded on these nanoplatelets growth of the FM phase will continue. Notice the similarities of this broad general behavior observed to the avalanche type processes seen in martensitic transitions as discussed in reference [18]. 

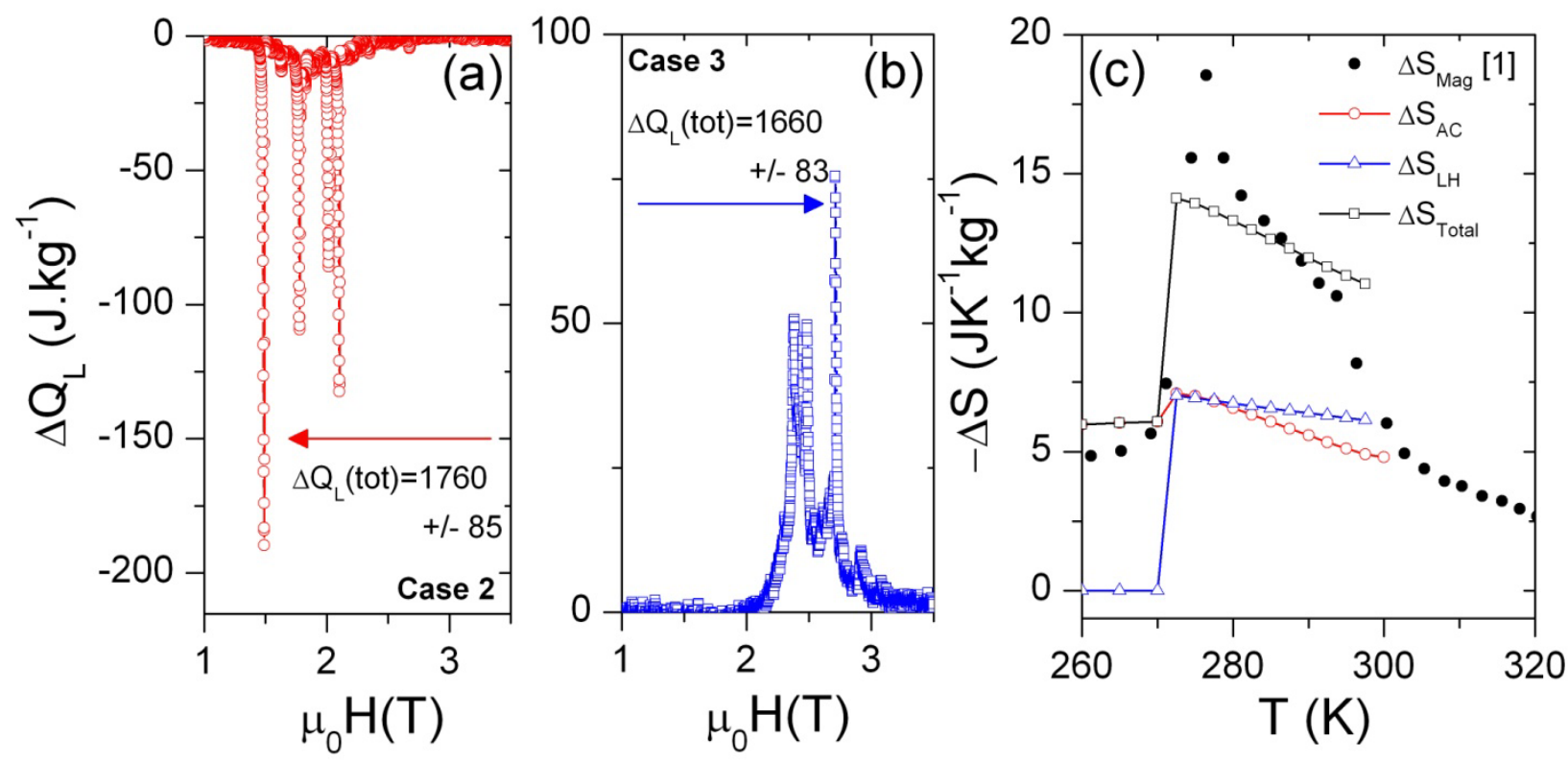

Figure 3. (a) \& (b) Close-up of the latent heat at $285 \mathrm{~K}$. (a) On field decrease the transition is nucleation dominated; there is no feature to seed the phase transition. (b) On field increase the transition is growth dominated; a second phase of $\mathrm{Gd}_{5} \mathrm{Ge}_{1.5} \mathrm{Si}_{1.5}$ nucleates the FM phase after which growth of the phase continues about this point. Total latent heat obtained by integrating the signal on demagnetization (a) and on magnetization (b) is comparable (within error). (c) Entropy change contributions from microcalorimetry compared to $\Delta S$ determined from magnetometry, $\Delta \mathrm{S}_{\mathrm{Mag}}{ }^{1}$ for a field change of 0-5T.

Figure 3(c) shows the entropy contributions determined from microcalorimetry compared to data taken from magnetometry of the bulk (taken from ref 1). Notice three things. Firstly there is reasonable agreement between the literature values and $\Delta \mathrm{S}_{\text {Total }}$ estimated from microcalorimetry over most of the temperature range. Secondly, the entropy contribution from latent heat is approximately $50 \%$ of the total entropy change, which is comparable to the contribution estimated from the structural component of $40-60 \% .{ }^{4,6}$ Lastly, as the temperature is increased (and the critical field, $\mathrm{H}_{\mathrm{c}}$ increases) there is only a small change in $\Delta \mathrm{S}_{\mathrm{LH}}$, i.e. the field and temperature accessible here does not significantly broaden the transition (or decouple the magnetic and structural components).

\section{CONCLUSIONS}

The different manifestations of latent heat in single crystal $\mathrm{Gd}_{5} \mathrm{Si}_{2} \mathrm{Ge}_{2}$ have been observed and correlated to Hall imaging reported previously. It was shown that in this system the impact of nanoplatelet inclusion is a lowering of the critical field that promotes the growth process of the first order phase transition. An increase in the growth process manifests as a broader latent heat response with respect to magnetic field. The total latent heat is determined by integrating the signal with respect to the field change direction and the difference between the two was shown to be comparable within error. It is also encouraging that the total entropy change from microcalorimetry compares well to values found in the literature. The latent heat observed in a $50 \times 100 \times 100 \mu \mathrm{m}$ fragment suggests a correlation length of approximately $20 \mu \mathrm{m}$, similar to the lengthscale suggested by Hall imaging. 


\section{ACKNOWLEDGEMENTS}

Work at Ames Laboratory is supported by the U.S. Department of Energy, Office of Basic Energy Sciences, Materials Sciences and Engineering Division. The Ames Laboratory is operated for the U.S. Department of Energy by Iowa State University under Contract No. DEAC02-07CH11358.

The authors would like to thank D.L. Schlagel (Ames Laboratory) for providing the single crystal $\mathrm{Gd}_{5} \mathrm{Si}_{2} \mathrm{Ge}_{2}$ sample.

\section{REFERENCES}

${ }^{1}$ V.K. Pecharsky and K.A. Gschneidner, Jr., Phys. Rev. Lett. 78, 4494 (1997)

${ }^{2}$ V.K. Pecharsky, and K.A. Gschneidner, Jr., Adv. Mater. 13, 683 (2001)

${ }^{3}$ L. Morellon, P. A. Algarabel, M. R. Ibarra, J. Blasco, B. García-Landa, Z. Arnold, and F. Albertini, Phys. Rev. B 58 R14721 (1998)

${ }^{4}$ G. J. Liu, J. R. Sun, J. Lin, Y. W. Xie, T. Y. Zhao, H. W. Zhang, and B. G. Shen, Appl. Phys. Lett. 88, 212505 (2006)

${ }^{5}$ L. Morellon, Z. Arnold, C. Magen, C. Ritter, O. Prokhnenko, Y. Skorokhod, P.A. Algarabel, M.R. Ibarra, and J. Kamarad, Phys. Rev. Lett. 93, 137201 (2004)

${ }^{6}$ V.K. Pecharsky, K.A. Gschneidner, Jr., Ya. Mudryk, D. Paudyal, J. Magn. \& Magn. Mater. 321, 3541-3547 (2009)

${ }^{7}$ H. Tang, A.O. Pecharsky, D.L. Schlagel, T.A. Lograsso, V.K. Pecharsky, and K.A. Gschneidner, Jr., J. Appl. Phys. 93, 8298 (2003)

${ }^{8}$ J.D. Moore, K. Morrison, G.K. Perkins, D.L. Schlagel, T.A. Lograsso, K.A. Gschneidner, Jr., V.K. Pecharsky, and L.F. Cohen, Adv. Mater. 21, 1-4 (2009)

${ }^{9}$ Y. Saruyama J. Therm. Anal. 38, 1827-33 (1992)

${ }^{10}$ A.A. Minakov, S.B. Roy, Y.V. Bugoslavsky, and L.F. Cohen, Rev. Sci. Instrum. 76, 043906 (2005)

${ }^{11}$ Y. Miyoshi, K. Morrison, J.D. Moore, A.D. Caplin, and L.F. Cohen Rev. Sci. Instrum. 79, 074901 (2008)

${ }^{12}$ V.K. Pecharsky, and K.A. Gschneidner, Jr., J. Appl. Phys. 90, 4614 (2001)

${ }^{13}$ S.B. Roy, G.K. Perkins, M.K. Chattopadhyay, A.K. Nigam, K.J.S. Sokhey, P. Chaddah, A.D. Caplin, and L.F. Cohen, Phys. Rev. Lett. 92, 147203 (2004)

14 J.D. Moore, G.K. Perkins, Y. Bugoslavsky, L.F. Cohen, M.K. Chattopadhyay, S.B. Roy, P. Chaddah, K.A. Gschneidner, Jr., and V.K. Pecharsky, Phys. Rev. B 73, 144426 (2006)

${ }^{15}$ K. Morrison, J.D. Moore, K.G. Sandeman, A.D. Caplin, and L.F. Cohen, Phys. Rev. B 79, 134408 (2009)

${ }^{16}$ G.K. Perkins, J.D. Moore, M.K. Chattopadhyay, S.B. Roy, P. Chaddah, V.K. Pecharsky, K.A. Gschneidner, Jr., and L.F. Cohen, J. Phys:. Condens. Matter. 19, 176213 (2007)

${ }^{17}$ F.J. Pérez-Reche, E. Vives, L1. Mañosa, I. Ràfols, R. Pérez-Magrané, and A. Planes, Phys. Rev. Lett. 87, 195701 (2001)

${ }^{18}$ Francisco-José Pérez-Reche, Marcelo Stipcich, Eduard Vives, Lluís Manõsa, Antoni Planes, and Michel Morin, Phys. Rev. B 69, 064101 (2004)

${ }^{19}$ A.O. Pecharsky, K.A. Gschneidner, and V.K. Pecharsky, J. Magn. \& Magn. Mater. 267, 60-68 (2003) 